\title{
Bedside ultrasound diagnosis of portal vein thrombosis following cholecystectomy: a case report
}

\author{
ROBERT BARONICA, TAJANA ZAH BOGOVIĆ, DRAŽENA GERBL, IVONA HANŽEK, DANIELA \\ BANDIĆ PAVLOVIĆ, VESNA VEGAR BROZOVIĆ, MLADEN PERIĆ \\ University Department of Anaesthesiology, Reanimatology and Intensive Care, University Hospital Centre Zagreb
}

Corresponding author:

Robert Baronica

University Department of Anaesthesiology, Reanimatology and Intensive Care,

University Hospital Centre Zagreb,

Kišpatićeva 12, 10000 Zagreb, Croatia,

Phone: +38512367858

E-mail: rbaronica@gmail.com

\section{ABSTRACT}

Portal vein thrombosis is a condition when the thrombus is blocking or narrowing blood flow of the portal vein. The initial approach in diagnosis of portal vein thrombosis for a non-transportable critically ill patient is a colour Doppler ultrasonography. We present a case of an 82 -year-old female with partial portal vein thrombosis following urgent cholecystectomy and choledochotomy. The clinical deterioration of the patient with hemodynamic and respiratory instability, acute renal failure, liver damage and metabolic acidosis, prevented the patient's transport for computed tomography diagnostics. A bedside abdominal ultrasonography was performed and revealed a partial obstruction of the left branch of the portal vein, while a confluent part of the portal vein showed a complete absence of flow. Therapy with low molecular weight heparin was immediately started. Definitive confirmation of portal vein thrombosis with the abdominal computed tomography imaging was possible almost 24 hours after clinical and laboratory deterioration. This case illustrates the importance of rapid bedside ultrasonography in diagnosis of thromboembolic events in the abdomen.

Key words: bedside ultrasonography, portal vein thrombosis, liver dysfunction

\section{INTRODUCTION}

Portal vein thrombosis (PVT) is a condition when the thrombus in a portal vein causes a partial or total obstruction to the blood flow. It is a relatively uncommon sur- gical complication, reported in literature mostly after laparoscopic procedures, liver transplantation or splenectomy. $(1,2)$ Other causes include cirrhosis, neoplasms, infections, and myeloproliferative disorders. (3) Although a diagnosis of PVT or thrombosis of other abdominal vessels is obtained by an abdominal computed tomography (CT) scan, the initial approach should be a Doppler ultrasonography. (4) In detecting PVT, colour Doppler sensitivity and specificity ranges from $89 \%$ to $93 \%$ and $92 \%$ to 99\% respectively. CT scan shows similar results with the advantage of more accurate display of the portal vein anatomy. (5)

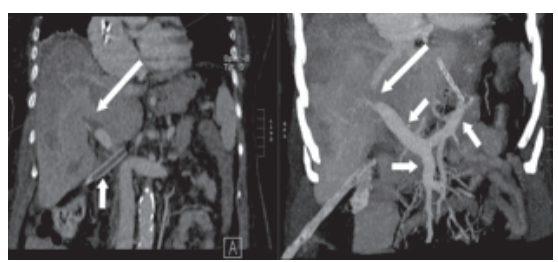

Figure 1. Computed tomography scan in portal venous phase. Long arrow is showing portal vein thrombosis. Short arrows are showing regular flow of splenic vein and superior mesenteric vein up to bifurcation of portal vein and $\mathrm{T}$ drainage after cholecystectomy and choledochotomy. Unfortunately, in the medical history of the patient there is only written ultrasound description and not the image itself.

\section{CASE REPORT}

An 82-year-old female was admitted to the Intensive Care Unit (ICU) following urgent cholecystectomy and choledochotomy, due to acute cholecystitis and choledocholithiasis. Her past medical history included stabile angina, hypertension, three coronary artery bypass grafts and one stent. Her usual therapy included bisoprolol, isosorbide mononitrate, trimetazidine dihydrochloride, perindopril, acetylsalicylic acid and simvastatin. At the time of admission in the ICU she was hemodynamically stabile. Eight hours later she became hypotensive, anuric and hypoglycemic. Laboratory tests revealed metabolic acidosis, elevated liver function tests and a significant increase of lactates and ddimers. Therapy was supportive and aimed towards the correction of single parameters. Since there was no improvement to volume resuscitation, a continuous administration of noradrenaline was started. On the chest $\mathrm{x}$-ray, slight left pleural effusion was noticed. In the 12 hours interval, laboratory tests showed significant increase of alanine aminotransferase (ALT) $4568 \mathrm{U} / \mathrm{L}$, aspartate aminotransferase (AST) 12070 $\mathrm{U} / \mathrm{L}$, alkaline phosphatase $664 \mathrm{U} / \mathrm{L}$, lactate dehydrogenase (LDH) $7390 \mathrm{U} / \mathrm{L}$ and bilirubin $140 \mu \mathrm{mol} / \mathrm{L}$. The platelet count was reduced $(52 \times 109 / \mathrm{L})$, the prothrombin time $(0.20 \%)$ and activated partial thromboplastine time (49.6s) prolonged and ddimers increased (>10 mg/L). Severe metabolic acidosis ( $\mathrm{pH} 7.18, \mathrm{BE}-15.4, \mathrm{HCO} 3$ $11.7 \mathrm{mmol} / \mathrm{L}$, lactate $10.49 \mathrm{mmol} / \mathrm{L}$ ) was present as well. We suspected the patient's sudden clinical deterioration (with laboratory signs of liver damage) was caused by hepatic blood flow obstruction. A radiologist was consulted and bedside ultrasound imaging was made immediately. A colour Doppler detected flow in the left branch of the portal vein, while the confluent part of the portal vein showed a complete absence of flow. The presence of some flow downstream from the thrombus was in- 
dicative to the partial PVT. At that time, the patient was too unstable for transport and a definitive diagnosis by CT angiography. A therapeutic dose of low molecular weight heparin (LMWH) was immediately started. During the second postoperative day, her laboratory tests revealed aggravated liver damage (ALT $8320 \mathrm{U} / \mathrm{L}$, AST 24100 U/L, LDH 13170 U/L, bilirubin 159 $\mu \mathrm{mol} / \mathrm{L})$. Despite generous volume therapy and diuretic stimulation, she was anuric, and continuous haemodialysis was necessary. At that moment, her hemodynamic status improved and a CT angiography was performed. A partial thrombosis of the portal vein, complete occlusion of the left portal branch and partial thrombosis of intrahepatic branches were found (figure 1). Further diagnostic procedures showed low levels of antithrombin III (AT III) (29\%, range $75.0-125.0 \%)$, protein $\mathrm{C}(26.4 \%$, range $70.0-140.0 \%)$ and protein $S(45.2 \%$, range $48.0-120.0 \%)$. In consultation with a haematologist, AT III replacement therapy was started and therapy with LMWH was continued. Soon, the patient's hemodynamic, respiratory and renal functions recovered, as well as coagulation and liver function tests. A control CT showed good conductivity in the main branch of the portal vein and hepatic veins. The patient was discharged to another ICU, where after few days biliary fistula and obstruction of choledochus were detected. Her clinical condition eventually deteriorated again, and she died of multiple organ failure.

\section{DISCUSSION}

Bedside ultrasonography in the ICU is an extremely valuable and sometimes underrated tool in abdominal vascular occlusions. (6) Severe hemodynamic instability and respiratory insufficiency in this group of patients, often delay definitive diagnosis and initiation of adequate therapy. In our patient, a definitive confirmation of PVT with the abdominal CT imaging was possible almost 24 hours after clinical and laboratory deterioration. Delay in the LMWH administration in therapeutic doses could increase the thrombotic occlusion of portal vein and other abdominal vessels or initiate pulmonary embolism. Initial differential diagnosis included ischemic hepatitis, acute coronary syndrome, pulmonary embolism, septic shock, aortic dissection and abdominal bleeding. The different therapy approach in these conditions and the dramatic clinical deterioration of our patient emphasise the importance of early bedside ultrasound diagnosis. Protein C, S and AT III deficiencies, that were later detected, may represent inherited disorders associated with PVT. (7) However, in the presence of liver damage, these proteins may be reduced secondary to the liver dysfunction. (8) No apparent cause of PVT is identified in more than $25 \%$ of patients. (7) Before ther is any evidence of ischemic liver injury, hemodynamic instability may be clinically apparent. (9) Such was the characteristic of our patient as well. A rapid rise in AST, ALT and LDH levels are typical findings in ischemic liver injury. (9) In our case, a severe elevation of liver func- tion tests was reached within the interval of eight hours, after the acute PVT caused hemodynamic disturbance with hypotension. After hemodynamic stabilization, liver function tests levels subsequently declined within seven to ten days. A treatment option in PVT includes anticoagulation with heparin to prevent extension of the clot, to allow recanalization and to prevent portal hypertension or intestinal infarction. $(4,5)$ There is an option for surgical thrombectomy, but in comparison to anticoagulation therapy alone, this procedure is not more efficient. $(4,8)$ Limited data suggest that patients diagnosed with acute PVT are unlikely to spontaneously recanalize their portal vein. (4) However, retrospective studies have found that in patients who were treated with anticoagulants, at least a partial recanalization occurs in $63-93 \%$ of cases. Complete recanalization occurs in $34-45 \%$ of cases. (4) In our case, therapeutic doses of LMWH attained the desired outcome, which was confirmed by the control CT scan.

\section{CONCLUSION}

This case demonstrates that in a critically ill patient a prompt bedside ultrasound diagnosis of PVT or other abdominal thromboembolic events may initiate appropriate therapy at the right time. It is an excellent diagnostic choice that may improve clinical outcome and possibly shorten ICU and hospital stays.

\section{REFERENCES}

1. James AW, Rabl C, Westphalen AC, Fogarty PF, Posselt AM, Campos GM. Portomesenteric venous thrombosis after laparoscopic surgery: a systematic literature review. Arch Surg 2009 Jun;144(6):520-526.

2. Ikoma N, Anderson CL, Ohanian M, Juneja HS, MacFadyen BV, Shah SK, KS Bajwa Portal Vein Thrombosis After Laparoscopic Cholecystectomy. JSLS 2014; 18(1):125-127.

3. Sacerdoti D, Serianni G, Gaiani S, Bolognesi M, Bombonato G, Gatta A. Thrombosis of the portal venous system. J Ultrasound 2007; 10(1):12-21.

4. Sanyal AJ. Acute portal vein thrombosis in adults: Clinical manifestations, diagnosis, and management. Editors: Chopra S, Travis AC. Aug 28, 2013. Retrieved from http://www.uptodate.com

5. Thomas RM, Ahmad SA. Management of acute post-operative portal venous thrombosis. J Gastrointest Surg 2010;14(3):570-577.

6. Chacko J, Brar G. Bedside ultrasonography-Applications in critical care: Part II. Indian J Crit Care Med 2014;18:376-381.

7. Sanyal AJ. Epidemiology and pathogenesis of portal vein thrombosis in adults. Editors: Chopra S, Travis AC. Sep 26, 2014. Retrieved from http://www.uptodate.com

8. DeLeve LD, Valla DC, Garcia-Tsao G. American Association for the Study Liver Diseases. Vascular disorders of the liver. Hepatology 2009;49(5):1729-1764.

9. Friedman LS. Ischemic hepatitis, hepatic infarction, and ischemic cholangiopathy. Editors: Chopra S, Travis AC. Dec 16, 2013. Retrieved from http://www.uptodate.com 